$\begin{array}{ll}\text { le portiQue } & \text { Le Portique } \\ \text { Revue de philosophie et de sciences humaines }\end{array}$

$18 \mid 2006$

Heidegger. La pensée à l'heure de la mondialisation

\title{
La pensée de Victor Frankl et notre temps
}

\section{Rolf Kühn}

\section{OpenEdition}

Journals

Édition électronique

URL : https://journals.openedition.org/leportique/833

DOI : 10.4000/leportique.833

ISSN : $1777-5280$

\section{Éditeur}

Association "Les Amis du Portique"

Édition imprimée

Date de publication : 1 septembre 2006

ISSN : 1283-8594

\section{Référence électronique}

Rolf Kühn, «La pensée de Victor Frankl et notre temps », Le Portique [En ligne], 18| 2006, mis en ligne le 15 juin 2009, consulté le 20 février 2023. URL : http://journals.openedition.org/leportique/833 ; DOI : https://doi.org/10.4000/leportique.833

Ce document a été généré automatiquement le 20 février 2023.

Tous droits réservés 


\title{
La pensée de Victor Frankl et notre temps
}

\author{
Rolf Kühn
}

1 Il y a un siècle, Vienne était, à côté de Paris et de Berlin, une capitale célèbre de l'Europe culturelle. Dans toutes les disciplines, des ruptures avec la tradition des convictions et valeurs s'y produisaient, comme par exemple la sécession esthétique avec Klimt, la musique nouvelle avec Schönberg, l'architecture fonctionnel avec Loos ou l'installation d'une nouvelle pensée anti-métaphysique par le Cercle Logique de Vienne (Carnap, Wittgenstein) d'une part et de la psychanalyse d'autre part ${ }^{1}$. Victor Frankl est donc né à une époque, comparable à la nôtre, où les valeurs du passé perdent leur évidence pour faire place à des "philosophies du soupçon» (Paul Ricœur) qui n'admettent plus rien d'autre que la vérification empirique ou une herméneutique du sens rongée par les incertitudes de l'inconscient. Pour le dire en un mot, lorsque Frankl publia ses premiers articles, le climat intellectuel et moral de l'époque est gouverné par le positivisme, naturalisme et psychologisme. C'est seulement en littérature que se manifeste encore un certain spiritualisme, comme chez Kafka ou Musil, où l'homme quête un Absolu lointain, c'est-à-dire poussé par l'angoisse de son existence qui se déroule hors d'une patrie à jamais perdue.

1. Positivisme scientifique et critique phénoménologique

2 Or, dès ses débuts, on trouvera chez Frankl trois concepts-clés : logos, éros et éthos, qui définissent, à la fois sur les plans philosophique et psychothérapeutique, ce qu'il appelle, à ce moment déjà, la " vocation » ou la «mission » de chaque individu ${ }^{2}$. C'est une décennie plus tard, surtout après l'expérience cruciale des camps de concentration, que Frankl va préciser ses premières intuitions par les concepts plus élaborés de l'analyse existentielle et de la logothérapie. On ne peut pas affirmer que Frankl a découvert tout seul cette nouvelle conception d'une psychologie entièrement humaniste, car il a reconnu lui-même, au moins deux prédécesseurs qui lui ont légué l'importance philosophique de la valeur à travers les donnés d'une conscience morale ou d'une responsabilité libre. 
3 Le premier à nommer est Rudolf Allers qui avait montré, contre Freud, que la conscience représente plus qu'un simple sur-moi soumis à la détermination de la socialisation familiale et culturelle, puisque je peux me décider, en toute liberté, contre n'importe quelle norme reçue, si une expérience authentique de ma «personne intime " l'exige dans son indépendance foncière ${ }^{3}$. Et cette réalité d'un être personnel infrangible est également à la base de la phénoménologie de Max Scheler qui avait élaboré, dès les années vingt, une philosophique ontologique des valeurs qui impliquent toujours un sens affectif et noétique en dehors du moi seulement empirique et historique ${ }^{4}$.

4 Nous nous rendons compte, immédiatement, qu'avec ces notions de conscience libre et de sens axiologique, Viktor Frankl possédait les instruments conceptuels nécessaires pour contrecarrer le psychologisme et le naturalisme positivistes de son temps. Dans ses conférences, ou dans des causeries privées à Vienne, il aimait faire allusion à un dessin qu'il avait fait pendant cette période de recherche et de rupture intellectuelles, et cela sous forme de son humour habituel : lui-même, ayant la taille d'un nain, se dresse sur les épaules de Freud et d'Adler comme personnages superposés. Par cette anecdote imagée, il s'avère clairement que Frankl a conçu «la troisième École psychothérapeutique de Vienne ", à savoir la sienne, aussi comme suite critique de la psychanalyse freudienne et d'une "psychologie herméneutique» adlérienne (verstehende Psychologie avant le titre plus tardif de Individualpsychologie). Car, si Adler s'était séparé de Freud, en 1911, à cause d'une interprétation plus descriptive ou phénoménologique de la névrose, Frankl fit de même à l'égard du mouvement adlérien vers la fin des années vingt. Pour lui, tout "arrangement névrotique " - en vue d'assurer la sécurité absolue d'une personnalité fictive - n'exclut pas qu'il y ait eu, au départ d'un tel vécu, un sentiment vrai. Par ce biais d'un tel vécu authentique au plus intime de la personne (echtes Erleben), Frankl se donnait donc le moyen conceptuel pour rejoindre, à la fois sur les plans thérapeutique et théorique, la réalité fondamentale d'une conscience vraie.

5 Il suffisait, afin de dégager l'existence concrète de n'importe quelle situation de la vie, d'interpréter la donation originaire et certaine des valeurs réelles par une phénoménologie adéquate de la "pré-compréhension ontologique du vécu existentiel » qui est propre à chaque individu avec sa valeur authentique. Par ce lien philosophique et psychothérapeutique, entrevu par Frankl grâce aux analyses de Scheler, Husserl et Heidegger ${ }^{5}$, il était en mesure de répondre, à sa manière d'une " pastorale médicale ", au défi que la phénoménologie classique avait alors lancé, de son côté, face au naturalisme scientiste régnant: à savoir que toute connaissance empirique ou théorique suppose, de prime abord, une subjectivité vivante ou une existence ouverte sur l'Être. Le Logos, l'Éros et l'Éthos du premier Frankl devint, par cette voie, l'intentionnalité du sens, la transcendance existentielle et le «Dieu inconscient » ou l'intuition axiologique de sa psychologie logothérapeutique ${ }^{6}$.

6 Le profond humanisme de Frankl ne repose alors, en fait, sur aucun choix théorique aveugle ou arbitraire, mais il s'enracine dans la meilleure tradition d'une philosophie critique et ontologique, afin de reprendre les question brûlantes du temps présent et de l'existence humaine dans leur origine même. Si, à la suite de sa détention comme juif voué à l'extermination, il écrit une pièce dramatique sur "Buchenau » (allusion aux camps réels de Buchenwald et d'Auschwitz), où il fait dialoguer les grandes figures philosophiques de Socrate, Spinoza et Kant au sujet de la réalité éthique vécue dans ces 
camps de concentration, il affirme par là directement la meilleure tradition grecque, hébraïque et chrétienne pour affronter l'horrible et le vide du monde totalitaire avec ses atrocités inimaginables. Pour cette raison, ses analyses ultérieures concernant la «frustration existentielle » dans une économie d'abondance, mais gouvernée par la seule technique et le capital, dépasse le seul constat psychothérapeutique d'une névrose généralisée, car il faut répondre, plus profondément, à un manque essentiel de nos sociétés qui sont marqués, en leur centre vital, par le soupçon rongeur et même l'impossibilité de tout sens, de toute identité vraie.

2. Les incertitudes du temps présent

7 Ainsi, si la logothérapie de Frankl, dès ses débuts viennois jusqu'à son extension internationale aujourd'hui, n'a pas perdu sa force initiale, c'est-à-cause de son lien crédible avec une vision inaltérable de l'homme et avec son approche phénoménologique de principe, comme il nous semble. Pour cette raison, analysons, en plus de cette "frustration existentielle " ${ }^{7}$ toujours existante, quelques d'autres traits de notre époque postmoderne, afin de voir, si le diagnostic de Frankl, médecin et penseur, s'avère toujours capable d'y apporter ses réponses. Tout d'abord, il faut constater que le psychologisme d'aujourd'hui n'est plus seulement un naturalisme qui s'oppose, idéologiquement, à toute conception philosophique ou religieuse de l'homme. Ce psychologisme comme croyance commune est devenu omniprésent, de telle façon qu'il commande presque entièrement les sciences humaines et leurs applications pratiques, et cela pour la simple raison de son alliance séculière avec la science technique. Par sa nature, cette dernière n'est pas psychologique, mais dans la mesure où la méthode galiléenne ou physique s'est imposée entretemps à tous les savoirs, la psychologie elle-même est devenue entièrement empirique, ou même empiriste. Elle s'applique, sans limite, aussi bien à la publicité qu'à la pédagogie scolaire et à la propagande politique pendant ou autour des conflits militaires, comme nous le voyons si bien actuellement en Irak. Cela veut dire que l'homme est conçu comme un simple «produit » biologique qui se trouve traversé par des motifs ou affects inconscients que l'on peut coordonner et orienter vers toute action souhaitable - soit bombardement aveugle ou soumission à tout ordre inhumain.

8 Il y a, pourtant, un domaine qui échappe à une telle idéologie ; c'est la souffrance sans remède, le destin sans recul pensable, même si l'on essaie également des stratégies psychologiques ou cognitives pour ces instants inéluctables. En suivant l'échelle des valeurs d'après Max Scheler, c'est-à-dire en affirmant l'axiologie originaire d'actes créatifs et esthétiques, Victor Frankl prévoit thérapeutiquement un changement de regard même dans les situations extrêmes de douleur et d'incapacité d'agir. Si je vis une telle situation où il m'est impossible de transformer encore les conditions de faits qui la composent, je peux modifier du moins mon attitude intérieure face à l'inaltérable. De cette manière, je transforme aussi ma nature psychique pour découvrir une dernière valeur personnelle ou existentielle : la valeur d'une telle attitude morale ou spirituelle en tant que telle, que Frankl nomme, en allemand, Einstellungswert, n'est pas une résignation, mais une force vivante ultime, puisqu'elle émane de ma liberté la plus profonde.

9 Par conséquent, si l'on analyse phénoménologiquement une telle situation, qui peut m'arriver à tout moment, on trouve ce qu'aucune stratégie psychologique ne peut donner, à savoir la source de ma vie comme telle. Il ne s'agit plus de la vie biologique ici, qui est une abstraction théorique des sciences physico-chimiques ou médicales. Il 
s'agit, au contraire, de cette vie phénoménologique pure ou absolue dont dépend tout phénomène, tout vécu et aussi toute valeur. Car pour apparaître, tout phénomène en son existence individuelle pour ma conscience intentionnelle, suppose une vie originaire qui est mon être auto-affectif même. En réclamant donc possible, également dans des situations à désespérer, une dernière attitude libre et responsable Frankl indique, par là, que le psychologisme scientifique ou omniprésent, et qui veut tout nous expliquer, avec des data empiriques et scientifiques, nous fait oublier l'essentiel: le Fond infini même de notre être, autrement dit cette vie originaire à la base de toute existence. Et le danger éminent n'est pas seulement d'oublier un tel Fond, mais de ne plus l'admettre comme cette réalité qui constitue notre être véritable.

À partir de cette donnée phénoménologique pure ou dernière, on peut faire un second constat pour notre temps postmoderne. Car, en fait, il n'y a plus d'identité effectivement possible dans un monde qui ne se construit que par un sens chaque fois partiel et relatif. Et cette négativité différentielle de principe ne peut être dépassée, en dernière analyse, que par la vie phénoménologique pure. Cette elle qui me constitue en tant que ce « Moi » inaltérable que je suis à tout moment, c'est-à-dire comme ce Moi qui existe en tant que cette ipséité pour laquelle toute sensation, impression, émotion ou pensée est absolument individuelle. Cette individualité veut dire que je ne peux pas me défaire de ma sensibilité qui est le mode immédiat de mon être unique, autrement dit mon identité vivante première et ultime.

$11 \mathrm{Si}$, en effet, Frankl affirme la valeur personnelle de chaque situation et de tout agir, il souligne ainsi que l'identité de ma vie ne vient aucunement de l'extérieur, ni de la nature finalement ou de l'histoire biographique, mais que mon identité véritable se trouve liée à la manière spécifique d'éprouver mon existence dans ce sentiment unique même - et de me décider alors noétiquement et pratiquement en conséquence ${ }^{8}$. Ainsi, contre l'anonymat de la technique, des sciences et de la politique, il y a, pour l'analyse existentielle de Frankl, un Soi originaire chaque foi irremplaçable qui n'est pas livré à la fabrication d'une identité psycho-dynamique illusoire par images, puisque son identité authentique réside dans ma manière de me sentir en ma qualité de personne vivante même. Cela explique pourquoi l'humanisme réfléchi de Frankl a toujours refusé tout conformisme social ou toute soumission à une idéologie régnante qui nie intrinsèquement la valeur unique de chacun. Pour Frankl, ce n'est finalement qu'à travers cette vocation subjective assumée par chacun que puisse prendre forme, éventuellement, une unité universelle de l'humanité à l'avenir.

12 Cela amène à une troisième observation pour notre temps. Par souci d'impartialité dans la rencontre psychothérapeutique, la logothérapie de Frankl se veut garder libre de toute religion au sens d'une confession particulière qui relève de la conviction personnelle de chacun. Mais cela n'empêche pas que toute angoisse existentielle, toute relation avec autrui, comme par exemple l'amour, ou tout sentiment de culpabilité, réelle ou névrotique, se trouvent placés, en dernière analyse, face à la question d'un Absolu, d'une Valeur suprême ou d'un Sens ultime - nommés Dieu ou autrement, et devant lesquels je ressens ma responsabilité intime. Ce qui importe, ici, c'est que la logothérapie n'empêche pas la clarification noétique d'un tel questionnement et qu'elle n'y voit pas seulement le signe d'infantilisme, comme c'est le cas dans la théorie de Freud et de ses successeurs jusqu'à Jacques Lacan. Dans une telle négation psychanalytique ou structuraliste (sans supprimer une telle régression possible), il faut saisir l'obnubilation foncière généralisée dans notre modernité, à savoir l'illusion 
qu'elle se croit à l'origine de tout réel. En parlant d'un « Dieu inconscient » comme chiffre transcendant d'une intuition infinie et de notre conscience morale, Victor Frankl suggère que nous touchons, dans notre intimité la plus reculée affectivement, une Réalité insondable qui est la Force même de toutes nos intuitions et motivations vraies.

$\mathrm{Au}$ positivisme et matérialisme techniques de nos temps s'ajoute, pour une telle analyse proposée, alors un nihilisme sans bornes qui n'est pas seulement la négation de telle ou telle valeur spécifique, mais la destruction principielle de la possibilité même d'une axiologie vivante ou originaire. Dans le langage de Frankl, c'est l'abolition même d'une Transcendance infinie et personnelle dans laquelle je prends ma naissance pour être confronté à elle en toute décision vraiment libre. Car cette liberté liée à un Absolu transcendant signifie, forcément, les limites d'un monde seulement imaginé et interprété à la mesure de nos connaissances et pouvoirs qui ne peuvent, en aucun sens, répondre par leurs seuls moyens au mystère abyssal en nous. En fait, on ne se trompe pas en disant que la psychothérapie de Frankl au sens d'une logothérapie réfléchie est une pensée et une praxis infiniment ouvertes, car elle n'ignore aucun aspect de l'expérience humaine possible en sa totalité. C'est pour cette raison même que le titre de notre texte annonçait "la pensée de Frankl " et non seulement sa "technique » thérapeutique basée, en outre, sur la "déréflexion » et la "transcendance » conçues comme une "distanciation" intérieure possible par rapport aux conflits psychodynamiques, afin d'être en mesure de correspondre, plus adéquatement, à l'appel concret de toute situation. Cependant, il nous faut parler, pour conclure, de la «méthode» franklienne en un sens précis, c'est-à-dire en comparaison avec toute autre méthode anthropologique.

3. Méthode et praxis de l'analyse existentielle

D'après ce que nous avons dit, vie axiologique, existence transcendante ainsi que conscience intime et infinie constituent la réalité et le but de la pensée de Frankl pour tout individu placé en face d'une opinion moderne qui nie toutes ces assertions pour n'affirmer qu'un individualisme vague. Celui-ci ne désigne pas l'ipséité absolument individuée qui prend sa naissance unique dans la Vie phénoménologique pure, et seulement en elle, mais cet individualisme actuel ne vise que les " comportements " psychologiques et leurs choix fortuits selon les circonstances. La psychologie et la psychothérapie de nos jours ne connaissent, en général, qu'une liberté arbitraire, de surface, qui suit, plus ou moins docilement, les impératifs publicitaires de nos sociétés et politiques pour satisfaire les besoins souvent artificiels d'une consommation dépendant d'un marché dit « libre » ou «global». Or, Victor Frankl avait une autre conception de la psychologie et de la psychothérapie. On la reconnaît très nettement dans son credo psychiatrique souvent souligné, dans ses écrits, et qui signifie que l'esprit ou le noétique en nous ne peuvent jamais tomber malades ${ }^{9}$. Si tel est le cas, il faut en conclure que sa vision de l'homme, de chaque individu particulier, n'est pas basée sur une approche déficitaire, comme dans d'autres anthropologies psychologiques, sociologiques et culturelles, mais sur la réalité d'une personne au contraire, qui est apte, à tout moment, d'une décision digne de l'appel d'absolu en elle. Par une telle perspective, tout homme (c'est-à-dire aussi le schizophrène et le névrosé par exemple) ne se trouve pas uniquement livré au seul régime de ses affects, hallucinations ou complexes, mais il demeure toujours en mesure de choisir aussi une attitude de grandeur face à son destin pulsionnel, psychosomatique ou extérieur. 

l'adaptation insuffisante de nos instincts à l'environnement où le désir primaire d'une satisfaction à assurer par une projection libidineuse ou fictive, méconnait ce pouvoir essentiel de l'homme de refuser son adhésion à une simple situation de faits constatables. Un tel refus, au nom du logos franklien, n'obéit pas à la négation de la réalité extérieure et intérieure ou de la coopération avec d'autres hommes, mais il constitue l'affirmation la plus directe de l'esprit qui ne peut être assimilé, à cause de son essence même, à aucune factualité visible. Si Frankl reprend ici l'enseignement fondamental de Max Scheler, parlant justement de la force incomparable de notre esprit de pouvoir dire «Non » aux circonstances matérielles, biologiques et psychiques du destin, il établit ainsi la praxis logothérapeutique sur une capacité illimitée de l'individu, comme on la trouve, d'une autre manière également, dans le doute hyperbolique de Descartes. Cette capacité de s'opposer et de résister pour l'esprit (Trotzmacht des Geistes) est illimitée, puisque ses racines plongent dans ce «Dieu inconscient» qui représente la Force chaque foi renouvelée pour transcender les donnés des situations et d'en faire une "tâche» individuelle confiée à la seule responsabilité de tel ou tel individu.

16 Autrement dit, plus loin que le déficit apparent d'une nature somatique ou psychique, il faut savoir reconnaître les potentialités inaltérées d'un «Je-Peux » phénoménologique originaire, comme le faisait Husserl, pour donner une forme différente à ce qui s'offre, au départ, seulement comme arrêt ou obstacle à notre action. Par ailleurs, il est intéressant à noter que la recherche actuelle sur les traumatismes, soit à la suite des viols fréquents, affrontements meurtriers ou des cataclysmes naturels, découvre dans le sujet traumatisé même une force d'auto-guérison, appelée "ressource» ou « résilience» toutes subjectives et individuelles ${ }^{10}$. Ce phénomène corrobore, en outre, la conviction philosophico-psychiatrique de Victor Frankl que l'essence même de notre existence vivante reste toujours indemne pour pouvoir être éveillée et soutenue lors d'une thérapie indiquée.

L'analyse existentielle, avec sa praxis logothérapeutique en tant que méthode psychologique, contredit, à ce moment, toute spéculation comme celle de Jacques Lacan par exemple, qui disait que toute affirmation d'un « Je » signifie, au fond, toujours : « Je suis un autre». Cette altérité à soi qui est le produit théorique d'un structuralisme psychanalytique à partir du système généralisé du langage avec son caractère de «Différence » principielle, conçoit l'homme comme un projet imaginaire balançant entre son désir pulsionnel et la facticité de ce qui est réellement. Dire «Je ", au sens de Lacan, ne voudrait signaler, en fin de compte, que la reconnaissance nécessaire de la réalité changeante ainsi que de mon moi toujours différent, afin de me réconcilier avec cet « Autre » que je suis à chaque fois d'une manière qui m'est étrangère.

$\mathrm{Si}$, en revanche, je situe l'identité véritable dans l'auto-affection de ma vie ou dans l'acte transcendant de la personne intime avant tout désir particulier, on peut affirmer et vivre une identité qui n'est pas la chimère d'un manque, mais une force ou un pouvoir originaires dont l'actualisation possible ne fait jamais défaut. Concrètement, l'analyse existentielle comme procédé logothérapeutique implique, par suite, une analyse phénoménologique de toutes les dimensions de notre être (corps, esprit et âme), pour saisir l'« ontologie dimensionnelle » d'une personne avec sa biographie en vue d'une unité vivante où le sens à vivre constitue le sentiment même d'une d'elle existence possible. Le sentiment ou l'affect n'est donc jamais un déficit, même si leur 
réalité psychologique est peut-être d'abord vécue douloureusement, car par toute émotion vraie se dessine déjà un projet de vie unique que je peux saisir en son authenticité.

19 Finalement, il est alors permis d'affirmer que la méthode franklienne vise une nouvelle habitualité spontanée de vivre qui correspond à une certitude intérieure qui n'est plus distincte de l'immédiateté même de chacun de mes vécus. Nous retrouvons, à cet endroit, l'authenticité de la personne en sa motivation la plus profonde, car la certitude vraie de sentir ou intuitionner immédiatement le sens concret de ma vie et de son agir, dans le moment présent, dépasse toute évidence purement intellectuelle ou normative. Une telle certitude phénoménologique pure fait aussi comprendre pourquoi le noétique, l'esprit ou le logos, au sens de Frankl, ne signifient nullement la ratio ou la réflexion seulement théorique, mais la réalité pluri-dimensionnelle de l'homme. Cette pluri-dimensionnalité de notre être fait sa richesse ontologique même, ce qui fait saisir la vérité existentielle qu'il ne faut jamais nier aucun aspect de la vie humaine pour la vivre en sa plénitude.

Nous avons suffisamment souligné que ce bonheur d'une existence subjective ou individuelle n'exclut ni la responsabilité ni la souffrance, lorsque celles-ci se présentent à nous. Mais le refus d'un manque anthropologique ou même ontologique dès le départ de l'analyse existentielle implique que le bonheur entier reste donné potentiellement à tout moment, si l'on sait orienter le regard. Et si Victor Frankl pointe volontairement, dans ses textes, ce phénomène heureux, c'est que - à la suite d'un seul moment véritable de bonheur vécu dans notre vie - cette vie entière semble trouver sa justification et son sens unifié. Cependant, Frankl ne veut pas seulement faire ressortir l'importance générale et thérapeutique du sentiment esthétique, de la relation affective et de l'accomplissement éthique qui nous comble. Comme Kafka, il veut nous dire surtout qu'avec chaque "bouchée de nourriture terrestre ", nous goûtons également une «bouchée d'éternité ». Celle-ci n'est pas une consolation illusoire, mais la valeur phénoménologique même de tout sentiment existentiel véridique, nous révélant une profondeur insondable de la vie qui reste toujours plus riche que tout ce que nous pouvons prévoir et produire par nous-mêmes.

21 En ce sens, l'humanisme philosophique et pratique de Frankl est une foi sans dogme cette foi, dont Karl Jaspers disait que "le maximum est le minimum pour l'homme ", voulant affirmer par là, comme la logothérapie existentielle, que la profondeur de l'homme égale sa transcendance, puisque les deux se rejoignent dans l'Infini ou l'Absolu. Et ceci n'est pas un énoncé abstrait, mais une telle réalité forme le noyau de la rencontre et de l'expérience thérapeutiques en soi. Car une thérapie digne de ce nom doit être à la hauteur de toutes les possibilités de l'homme, si l'on ne veut pas tomber dans des réductionnismes meurtriers, comme le montre le désarroi de notre temps. En effet, l'invitation à la clairvoyance constitue toujours le cœur d'une pensée vraie et de sa praxis réelle qu'elle inspire. Je voudrais souhaiter à nous tous une telle lucidité sans morosité. 


\section{NOTES}

1.. Voir Y. PÉLICIER (éd.), Les Écoles de Vienne, Paris, 1988, avec une contribution sur Frankl de F. Da Fonseca : « Victor Frankl et la logothérapie », p. 185-191.

2.. Voir „Psychotherapie und Weltanschauung. Zur grundsätzlichen Kritik ihrer Beziehungen“, Internationale Zeitschrift für Individualpsychologie 3 (1925), p. 250-252 ; „Zur geistigen Problematik der Psychotherapie“, Zentralblatt für Psychotherapie 10 (1938), p. 33-45 ; „Philosophie und Psychotherapie. Zur Grundlegung einer Existenzanalyse“, Schweizerische Medizinische Wochenschrift 31 (1939), p. 707-709.

3.. Voir R. ALLERS, Das Werden der sittlichen Person, Fribourg-en-Brisgau, 1929 (trad. anglaise The Psychologie of Character, Londres, 1978). Voir aussi R. KÜHN, Sinn - Sein Sollen. Beiträge zu einer phänomenologischen Existenzanalyse in Auseinandersetzung mit dem Denken Viktor E. Frankls, Cuxhaven-Dartford, $3^{e}$ édition 1995, p. 100-134.

4.. Voir Der Formalismus in der Ethik und die materielle Wertethik. Neuer Versuch der Grundlegung eines ethischen Personalismus (Ges. Werke 2), Bern-Munich, $6^{\mathrm{e}}$ édition 1980 ; trad. française Le Formalisme et l'Éthique matérielle des valeurs, Paris, 1955.

5.. Au sujet de ce dernier, voir maintenant : Pascal LE VAOU, Martin Heidegger peut-il nous aider à lire Victor Frankl ? Le Logos entre herméneutique et déconstruction, in Dominik BAтTHYÁny/Otto zsoK (éds.), Viktor Frankl und die Philosophie, Vienne, Springer, 2005, p. 253-286.

6.. Voir, pour ses allusions, ces ouvrages : Ärztliche Seelsorge, Vienne, $10^{e}$ édition 1983 ; Der unbewußte Gott. Psychotherapie und Religion (en partie écrit en 1948), Munich, $6^{\mathrm{e}}$ édition 1985 ; trad. française Le Dieu inconscient, Paris, 1975.

7.. Voir surtout son texte „Der Wille zum Sinn und seine Frustration durch die moderne Industriegesellschaft", in Hemmende Strukturen in der heutigen Industriegesellschaft, Rüschikon-Zurich, 1969, p. 45-56.

8.. Voir aussi notre comparaison entre Simone Weil et Victor Fankl : « De la vie affective en philosophie et en psychologie ", Revue des sciences philosophiques et théologiques 4 (1985), p. 548-572; ainsi que Radicalité et passibilité. Pour une phénoménologie pratique, Paris, 2003, chap. II. 8 : « Existence et affectivité », p. 165-190, et aussi « La mélancholie est encore une bonne humeur », Actualités psychiatriques 1 (1995), p. 7-9. 9.. Voir V. FRANKL, „Grundriß der Existenzanalyse und Logotherapie“, in Handbuch der Neurosenlehre (édité par V. E. Frankl, V. E. Freiherr von Gebsattel, J. H. Schultz), MunichBerlin, 1959, p. 663-736.

10.. Voir, par exemple, Boris CYRULNIK, Un merveilleux malheur, Paris, Odile Jacob, 1999.

\section{RÉSUMÉS}

«La pensée de Victor Frankl et notre temps ». La pensée philosophique et logothérapeutique de V. E. Frankl est basée sur une phénoménologie de la liberté et du sentiment d'existence qui mettent en question les réductionnismes actuels du psychologisme, positivisme et nihilisme. 
L'individu réel répond à la vie à la fois axiologique et absolue pour donner un sens responsable à sa situation chaque fois unique.

"Victor Frankl's thought and our time". Victor E. Frankl's philosophical and logotherapeutical thought is based on a phenomenology of freedom and of the feeling of existence that puts to question the present reductionisms of psychologism, positivism and nihilism. The true being answers to life both axiologic and absolute to give a responsible meaning to his situation, each time unique.

"El pensamiento de Victor Frankl y nuestro tiempo". El pensamientoy logo terapéutico de Victor E Frankl arraiga en una fenomenología de la libertad y del sentimiento de existencia que cuestionan los reduccionismos actuales del psicologismo, positivismo y nihilismo. El individuo real responde a la vida a la vez axiológica y absoluta para dar un sentido responsable a una situación que es cada vez única.

"Viktor Frankl und unsere Zeit". Frankls Philosophie und Logotherapie lehnt sich an eine Phenomenologie der Freiheit und widersetzt sich jedem Psychologismus, jedem Positivismus, jedem Nihilismus.

\section{AUTEUR}

\section{ROLF KÜHN}

Rolf Kühn, né en Allemagne en 1944, est docteur en philosophie de l'Université ParisSorbonne. Il a obtenu une thèse d'État en philosophie de l'Université de Vienne en Autriche. Il est auteur et traducteur en philosophie à Fribourg-en-Brisgau, surtout dans le domaine de la phénoménologie actuelle ; outre les nombreuses publications en langue allemande, il a également publié en français : Radicalité et passibilité. Pour une phénoménologie pratique, L'Harmattan, 2003. Voir aussi : www.lebensphaenomenologie.at 